\title{
Carnets
}

Revue électronique d'études françaises de l'APEF

Première Série - 1 Numéro Spécial | 2009

Cultures littéraires : nouvelles performances \& développement

\section{La littérature française dans les programmes scolaires congolais. Un choix de développement culturel conflictuel}

\section{Omer Massoumou}

\section{OpenEdition}

Journals

Édition électronique

URL : http://journals.openedition.org/carnets/4235

DOI : $10.4000 /$ carnets.4235

ISSN : 1646-7698

Éditeur

APEF

Édition imprimée

Date de publication : 1 juin 2009

Pagination : 379-388

\section{Référence électronique}

Omer Massoumou, «La littérature française dans les programmes scolaires congolais. Un choix de développement culturel conflictuel », Carnets [En ligne], Première Série - 1 Numéro Spécial | 2009, mis en ligne le 16 juin 2018, consulté le 01 mai 2019. URL : http://journals.openedition.org/carnets/4235 DOI : $10.4000 /$ carnets. 4235

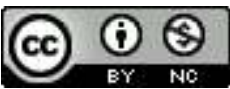

Carnets est mis à disposition selon les termes de la licence Creative Commons - Atribution - Pas d'utilisation commerciale 4.0 International. 


\title{
LA LITTERATURE FRANÇAISE DANS LES PROGRAMMES SCOLAIRES CONGOLAIS

\author{
Un choix de développement culturel conflictuel
}

\author{
OMER MASSOUMOU \\ Université Marien Ngouabi \\ omer.massoumou@gmail.com
}

\begin{abstract}
Résumé
L'objet de notre réflexion est d'étudier le rôle assigné à l'enseignement de la littérature au Congo dans les classes du secondaire littéraire (lycée). En considérant les choix institutionnels au sujet des œuvres littéraires, il apparaît que le Congo accorde une place non négligeable aux littératures congolaise et française. Nous construisons notre réflexion autour de trois axes. Nous allons premièrement aborder des questions liées aux éléments linguistiques, idéologiques, esthétiques ou thématiques motivant éventuellement le choix des textes et des genres littéraires. Nous voudrons deuxièmement apprécier le sens de la juxtaposition des deux littératures, nationale et française dans les programmes. Troisièmement nous traiterons d'une donnée diachronique. Pour le Congo le choix actuel des œuvres littéraires répond au contexte historique post-monopartiste (1990) où la censure n'est plus de mise. La notion de liberté qu'apporte la régime politique démocratique semble ouvrir la voie à toute valeur idéologique, culturelle et esthétique.
\end{abstract}

\section{Abstract}

The goal of this study is to demonstrate the role assigned to the literary teaching in the Republic of Congo in the fifth form of literature (secondary school). Considering the institutional choice of the literary works, it appears that the Republic of Congo attaches an important value to the Congolese and French literatures. Our study will bear on threefold key points. Firstly, we are going to deal with questions related to linguistic, ideological, aesthetic or thematic issues motivating the choice of the literary textbooks and genres. Secondly, we want to appraise the meaning of the juxtaposition of the national and French literatures in the curriculums. Thirdly, we will concentrate on diachronic data. As regards the Republic of Congo, the present choice of literary works refers to the post single party historical context (1990) when the censorship is not obligatory. The liberty notion advanced by the democratic political regime seems to open the way to ideological, cultural and aesthetic values.

Mots-clés: Littérature congolaise, Littérature française, Réception, Valeurs littéraires

Keywords: Congolese literature, French literature, Reception, Literacy values 


\section{Introduction}

L'objet de notre réflexion est de montrer comment le rôle assigné à l'enseignement de la littérature au Congo dans les classes du secondaire littéraire (lycée) ne répond pas à un objectif clair et à une véritable demande de formation. L'offre d'éducation définit un cursus où les compétences à acquérir ne coïncident pas à des attentes exprimées et restent peu suivies si nous tenons compte de la théorie de la réception de Hans Robert Jauss. Pour la formation de la personnalité de l'élève, les œuvres étudiées dans le cursus scolaire devraient jouer un rôle décisif. En considérant les choix institutionnels au sujet des œuvres littéraires, il apparaît que le Congo accorde une place non négligeable aux littératures congolaise et française. Nous nous sommes intéressé particulièrement au cursus des élèves de la série littéraire des lycées d'enseignement général. Nous relevons que les ouvrages au programme relèvent des littératures congolaise et française et qu'ils ne répondent pas explicitement à un horizon d'attente.

Une telle réalité, est-elle voulue ? Vise-t-elle une appropriation de la langue et de la culture française par la littérature? Explique-t-elle une volonté institutionnelle de rattachement à la France ? Le programme de français du lycée élaboré par l'Institut national de recherche et d'actions pédagogiques (INRAP, 2002) ne fournit en effet aucune réponse à ces différentes questions. Nous apprenons au début de ce programme que «le français est perçu comme un instrument pour aborder la littérature » (INRAP, $2002: 1$ ) et que les quatre objectifs généraux du programme portent sur la compréhension d'une œuvre, l'expression orale, l'expression écrite et le développement des compétences acquises.

Nous allons premièrement aborder des questions liées aux éléments linguistiques, idéologiques, esthétiques ou thématiques motivant éventuellement le choix des textes et des genres littéraires. II s'agit de traiter de la question de la réception institutionnelle qui est bien favorable à la littérature française dans le cas des programmes de français du secondaire deuxième degré, par ce fait même évaluer celle (la réception) des élèves. Nous voudrons deuxièmement apprécier le sens de la juxtaposition de deux littératures, nationale et française dans les programmes. Nous chercherons à montrer si cela repose sur un a priori favorable au sujet de la littérature française qui contribuerait à une meilleure éducation des apprenants. S'il apparaît que les acteurs du système éducatif semblent porter peu d'attention à l'histoire des textes littéraires retenus et réduisent l'approche du texte littéraire au fonctionnement narratif et à la morale de l'histoire, nous réalisons que ces objectifs ne correspondent pas au rôle que doit assumer une littérature surtout la littérature moderne et contemporaine. Nous présenterons quelques aspects conflictuels relevés dans l'enseignement de la littérature. Troisièmement nous traiterons d'une donnée diachronique. 
Pour le Congo, le choix actuel des œuvres littéraires répond au contexte historique postmonopartiste (1990) où la censure n'est plus de mise. La notion de liberté qu'apporte le régime politique démocratique semble ouvrir la voie à toute valeur idéologique, culturelle et esthétique. Toute œuvre semble maintenant accessible et cela pose également un problème. Nous présenterons les résultats d'une enquête mettant en exergue la divergence d'intérêt accordée par les élèves aux deux littératures enseignées.

Dans cette réflexion, nous voudrons porter spécifiquement notre attention au programme de la série littéraire.

\section{Une réception divergente de la littérature française}

La réception de la littérature française au Congo est assez contradictoire si l'on observe l'attitude des principaux acteurs du système éducatif. En effet, les organismes étatiques restent très favorables à la littérature française alors que les élèves du secondaire la rejettent majoritairement. Le tableau 1 relatif aux œuvres littéraires au programme dans les lycées d'enseignement général est un point de départ pour faire un commentaire sur les choix institutionnels dans les programmes de formation des élèves.

\begin{tabular}{|c|c|c|c|}
\hline & $\begin{array}{l}\text { CEuvres littéraires au } \\
\text { programme de la série } \\
\text { littéraire }(A)\end{array}$ & $\begin{array}{l}\text { CEuvres littéraires au } \\
\text { programme des séries } \\
\text { scientifiques (C et } D)\end{array}$ & $\begin{array}{l}\text { CEuvres littéraires au } \\
\text { programme de toutes } \\
\text { les séries }(A, C, D)\end{array}$ \\
\hline $\begin{array}{l}\text { Classes de } \\
\text { seconde }\end{array}$ & $\begin{array}{l}\text { - Chroniques } \\
\text { congolaises de Tati } \\
\text { Loutard } \\
\text { - L'Ecole des femmes de } \\
\text { Molière } \\
\text { - Dictionnaire français }\end{array}$ & $\begin{array}{l}\text { - Chroniques } \\
\text { congolaises de Tati } \\
\text { Loutard } \\
\text { - Dictionnaire français }\end{array}$ & $\begin{array}{l}\text { - Anthologie des } \\
\text { littératures } \\
\text { francophones (XVI et } \\
X V I I^{e} \text { siècles) }\end{array}$ \\
\hline $\begin{array}{l}\text { Classes de } \\
\text { première }\end{array}$ & $\begin{array}{l}\text { - L'Anté peuple de Sony } \\
\text { - Labou Tansi } \\
\text { - L'Etranger de Camus } \\
\text { - Anthologie de poésie } \\
\text { de J. Chevrier } \\
\text { - Dictionnaire français }\end{array}$ & $\begin{array}{l}\text { - L'Etranger de Camus } \\
\text { - Dictionnaire }\end{array}$ & $\begin{array}{l}\text { - Anthologie des } \\
\text { littératures } \\
\text { francophones (XVIII } \\
\text { et } X I X^{e} \text { siècles) }\end{array}$ \\
\hline $\begin{array}{l}\text { Classes de } \\
\text { terminale }\end{array}$ & $\begin{array}{l}\text { - Le Pleurer-rire de H. } \\
\text { Lopes } \\
\text { - Le Mariage de Figaro } \\
\text { de Beaumarchais } \\
\text { - Dictionnaire français }\end{array}$ & $\begin{array}{l}\text { - Le Pleurer-rire de H. } \\
\text { Lopes } \\
\text { - Dictionnaire français }\end{array}$ & $\begin{array}{l}\text { - Anthologie des } \\
\text { littératures } \\
\text { francophones ( XX } \\
\text { siècle) }\end{array}$ \\
\hline
\end{tabular}

Tableau 1 : les œuvres littéraires au programme au lycée 
Nous portons essentiellement notre attention sur le programme de la série littéraire. La formation littéraire est en effet envisagée à partir d'une œuvre congolaise et d'une œuvre française. La recommandation d'un dictionnaire de langue française conforte la référence à la France en tant que pays « fournissant » la langue et la littérature dans l'enseignement au Congo. Nous relevons en effet qu'en classe de seconde ce sont Les Chroniques congolaises de Jean-Baptiste Tati-Loutard et L'Ecole des femmes de Molière, en classe de première ce sont L'Anté-peuple Sony Labou-Tansi et L'Etranger d'Albert Camus et en terminale, ce sont Le Pleurer-rire d'Henri Lopes et Le Mariage de Figaro de Beaumarchais qui sont juxtaposés Cette juxtaposition établit une situation où les littératures congolaise et française apparaissent comme les principaux modèles pour la formation culturelle des jeunes congolais. Ces littératures deviennent des objets d'étude pour les jeunes qui n'ont pas nécessairement les prérequis pour bien assimiler les différentes composantes. L'organisme étatique, I'INRAP qui a retenu cette double dynamique ne la justifie pas. Nous pensons que pour les décideurs, la France, en tant qu'ancien pays colonisateur du Congo, elle continue de représenter un modèle de développement culturel, idéologique et esthétique. Ainsi, le Congo est situé de façon latente dans l'histoire littéraire ou culturelle de la France. Hans Robert Jauss affirme (1978 : 270) : «Pour qu'une œuvre du passé continue d'être agissante, il faut qu'elle suscite l'intérêt latent ou délibéré de la postérité qui poursuit sa réception ou en renoue le fil rompu ». Cette filiation latente correspond à une ouverture sur la richesse de la littéraire française où les genres, les mouvements, les idées, etc. permettent d'aborder la littérature congolaise et d'autres littératures nationales. Le caractère «classique » des œuvres de la littérature française abondamment étudiées favorise leur assimilation par le lecteur congolais. Les œuvres au programme permettent aussi de comprendre les genres littéraires : théâtre, roman, poésie...

Mais nous pensons que le choix des œuvres littéraires françaises est conflictuel et repose sur des approximations délicates. Nous avons relevé que pour l'INRAP, les objectifs pédagogiques suffisaient à justifier l'approche d'une pièce de théâtre ou d'un roman compris comme genre. Une telle façon de faire permet de parler d'une réception restrictive et cela relègue à un niveau négligeable des aspects sur l'histoire littéraire, sur l'évolution des idées culturelles, sur les aspects esthétiques, etc. Et comme les élèves ne sont pas préparés à lire ces ouvrages, ils les trouvent peu intéressants. En classe de seconde par exemple, la lecture de L'Ecole des femmes reste délicate si le texte est en ancien français. En plus, par son cursus, l'élève n'a pas appris la versification française. Si le thème du mariage peut intéresser des adolescents, mais le cadre esthétique est jugé ennuyeux. Les élèves vont davantage rire avec Trois prétendants un mari qu'avec L'Ecole des femmes. Nous voulons 
en effet dire que la réception des œuvres françaises par les élèves est plutôt une réceptionrejet. II n'existe pas de véritable dialogue entre l'élève congolais et le texte qui lui est proposé. Au secondaire, les élèves n'apprécient pas favorablement la littérature française. Ils préfèrent à $65,76 \%$ la littérature congolaise. La nécessité de dialogue dont parle $H$. R. Jauss est absente :

L'effet de l'œuvre et sa réception s'articulent en un dialogue entre un sujet présent et un dialogue passé ; celui-ci ne peut encore "dire quelque chose" à celui-là que si le sujet présent découvre la réponse implicite contenue dans les discours passés et l'aperçoit comme réponse à une question qu'il lui appartient, à lui, de poser maintenant. (Hans Robert Jauss, 1978 : 271).

Si l'enseignement de la littérature congolaise pose moins de problème parce que les textes nous sont contemporains et que les problèmes abordés sont souvent ceux que la société congolaise a connus, cela n'est pas le cas pour la littérature française.

Si nous admettons que chaque texte littéraire représente à la fois un espace linguistique, idéologique, culturel et esthétique, nous ne comprenons pas comment ici les textes littéraires offerts aux jeunes congolais ne sont pas abordés dans l'esprit de la diffusion des biens culturels.

\section{Le sens d'une juxtaposition littéraire}

Pour la série littéraire, nous l'avons observé, le programme d'enseignement repose sur une double dynamique. En posant la littérature française comme la principale littérature étrangère au programme, l'Etat congolais a défini une filiation, une ouverture à la culture française. II est évident que si à la place des auteurs français, le programme avait retenu des auteurs francophones (canadiens, belges) ou non francophones (chinois ou lusophones), la formation culturelle aurait une incidence non pas d'acceptation voire d'assimilation mais de découverte d'un autre espace culturel. II y aurait nécessairement une attitude différente. La forte présence de la littérature française replace dans le rapport historique du colon à colonisé, du maître et du valet, ce qui peut tendre à dénaturer l'esprit critique.

Mais nous sommes tenté de croire aussi que la place accordée à la littérature française correspond à une démarche de convenance, de facilité. II était en effet plus aisé pour les décideurs de choisir des auteurs français dont les nombreuses approches descriptives, thématiques en favorisaient la saisie. Faute de réponses institutionnelles, nous essayons de définir des hypothèses qui peuvent expliquer une telle réalité. On pourrait ainsi retenir que le fait que la France appuie probablement le système éducatif congolais et cela 
se traduit en retour par la réalité décrite. On pourrait aussi penser à un métissage culturel dans la mesure où les jeunes lycéens reçoivent un enseignement sur deux littératures basées sur des cultures différentes. Si par la littérature congolaise, les élèves restaient au contact des valeurs culturelles locales, par la littérature française, ils accédaient à un espace culturel de modernité.

Ces postulats qui peuvent avoir fondé la structuration du programme de la série littéraire nous permettent de penser ici la réception que les élèves réservent aux deux littératures au programme. Si au niveau institutionnel, les réponses officielles n'existent pas, pour ce qui concerne la réception des littératures par les élèves, il a juste été question de mener une enquête auprès d'une centaine d'entre eux pour évaluer le poids des littératures congolaise et française. Nous avons ainsi interrogé 111 élèves au niveau de deux lycées d'enseignement général à Brazzaville ${ }^{1}$. Sans chercher à reprendre ici les détails des questions, nous voulons relever quelques réponses qui donnent des indications sur les sentiments des élèves. L'une des questions portait sur la lecture jugée difficile ou facile des deux littératures. De façon majoritaire, $51 \%$ d'élèves ont jugé la littérature française d'accès difficile contre $28 \%$ pour la littérature congolaise (le reste des élèves ne s'étant pas prononcés). Si les élèves affirment connaître des auteurs français (Beaumarchais [cité par 96 élèves], Camus [80], Molière [75]), ce sont surtout des auteurs au programme. $66 \%$ des élèves affichent une préférence de la littérature congolaise. Ils trouvent en effet l'étude d'une œuvre littéraire congolaise ou africaine plus intéressante pour des raisons diverses dont l'univers culturel, les réalités sociopolitiques, le vocabulaire... Mais la préférence de la littérature n'est pas nécessairement traduite par une connaissance effective des textes ou des auteurs. Les auteurs congolais ou africains sont en effet curieusement moins cités comme auteurs lus. Le tableau suivant nous permet de relever les discordances.

\begin{tabular}{|l|l|c|}
\hline \multicolumn{1}{|c|}{ Genre } & \multicolumn{1}{c|}{ CEuvres } & $\begin{array}{c}\text { Nbre d'élèves } \\
\text { ayant lu le livre }\end{array}$ \\
\hline \multirow{2}{*}{ Romans } & L'Etat honteux & 2 \\
\cline { 2 - 3 } & L'Etranger & 45 \\
\hline \multirow{2}{*}{ Pièces de théâtre } & Mariage de Figaro & 25 \\
\cline { 2 - 3 } & Trois prétendants un mari & 11 \\
\hline \multirow{2}{*}{ Poésies } & Fleurs du mal & 10 \\
\cline { 2 - 3 } & Mauvais sang & 4 \\
\hline
\end{tabular}

Tableau 2 sur la lecture des œuvres

\footnotetext{
${ }^{1}$ L'enquête a été menée dans le cadre d'un programme qui vise à définir la réception de la littérature française au Congo. Une publication y relative est prévue en fin d'année ou au de l'année 2010.
} 
Il apparaît clairement que les élèves affirment avoir lu majoritairement les auteurs français. Si l'on peut légitimement se poser des questions sur le caractère intégral ou non de ces lectures, il y a tout de même une réalité contradictoire dans leurs affirmations. Peut-être que la circulation plus importante des œuvres de la littérature française en facilite l'accès ${ }^{2}$. Par ailleurs, l'absence d'études critiques envisageant une didactique des œuvres rend délicate la lecture de la littérature congolaise. Pour la littérature française, il existe des profils des œuvres, des études commentées et annotées, des résumés... disponibles et exploitables par l'enseignant, mais rien de tout cela pour la littérature congolaise. Le professeur de français n'a pas les outils didactiques pour penser les résumés des œuvres ; pour illustrer le fonctionnement narratif, dramaturgique ou poétique des textes comme les Chroniques congolaises, L'Anté-peuple, Le Pleurer-rire, etc.

\section{Un contexte politique d'incertitude (incidence politique)}

Le renouveau démocratique qu'apportent les changements de régime politique au début de la décennie 90 donne des orientations nouvelles dans le système éducatif. Après les indépendances (1960), le Congo connait une seconde indépendance avec des dénonciations des pratiques socialistes (1963-1991) et la proposition de nouvelles orientations démocratiques. L'avènement du pluralisme politique coïncide avec un élan d'ouverture sur le monde. Ainsi le rôle assigné à la littérature dans le système éducatif semble répondre, dans l'ensemble, à une dynamique de liberté et d'ouverture à l'altérité. Le fonctionnement de la littérature n'est visiblement pas ignoré dans le choix des textes et des auteurs.

en dehors des procès d'intention ou d'opinion que l'idéologie fait ça et là aux écrivains mal engagés - sans parler des vrais procès, dont les tenants et les aboutissants ne sont jamais du ressort de la littérature -, il apparaît plutôt que l'écrit, s'il est souvent jugé sur sa valeur de «message », est en lui-même hors de toute juridiction, de sorte que l'auteur, libre d'user et d'abuser du langage selon les fins qu'il se propose, reste assuré littérairement parlant d'une parfaite impunité (Marthe Robert, 1977 : 62).

Le choix des auteurs et d'ouvrages reste peu motivé mais il se redéfinit dans une dynamique de changement. Ce qui importe pour les décideurs, c'est le contenu sémantique

\footnotetext{
${ }^{2}$ Lors du colloque international de novembre 2008 sur Tchicaya U tam'Si, il a été constaté que les œuvres de ce poète congolaise étaient introuvables ni dans les librairies de Brazzaville, ni dans les bibliothèques, le centre culturel français y compris.
} 
du message. C'est dans cette phase de l'histoire nationale congolaise que des œuvres littéraires nouvelles ont été proposées au programme d'enseignement secondaire.

Jean-Baptiste Tati-Loutard, Sony Labou Tansi et Henri Lopes sont des auteurs phares de la littérature congolaise. Ils ont marqué l'histoire immédiate (années soixante-dix, quatre-vingts et quatre-vingt-dix). C'est sans nul doute leur position, leur envergure au niveau national en tant qu'acteurs socio-politiques et écrivains qu'ils bénéficient d'une véritable promotion. Les œuvres enseignées au lycée permettent aux élèves de se représenter les réalités congolaises. II s'agit de les situer dans une culture locale, celle que décrivent Les Chroniques congolaises dans sa légèreté quotidienne mais que présentent L'Anté-peuple et Le Pleurer-rire dans une évidente violence symbolique. Nous pensons que l'enseignement d'une littérature de violence après une période de séquestration de la liberté a eu pour principal avatar l'expression de la part sombre de l'être humain. Mais il est sans nul doute légitime de se poser des questions sur l'incidence des violences littéraires dans le basculement tragique des guerres civiles de la décennie quatre-vingt-dix. La littérature congolaise a rendu envisageable le crime de l'autre par une symbolisation devenue anodine de la guerre. S'il y a une évidente gravité, des faits relevés par les œuvres, la part de cette gravité ne semble pas suffisamment prise en compte par les acteurs sociaux.

Quant à la littérature française, elle propose une ouverture à un univers de modernité dont les œuvres retenues expriment les caractéristiques. Pour apprendre la littérature, il semble que le modèle incontestable reste celui de la littérature française. Le roman, le théâtre ou le poème sont compris dans la dynamique de l'histoire littéraire de la France. Le Congo s'inscrit dans la dynamique du passé français. Ainsi les élèves traitent des auteurs français des différents siècles, des notions de genre littéraire, de versification comme des vérités immuables. Les œuvres retenues continuent d'agir, de susciter l'intérêt des lecteurs congolais. Hans Robert Jauss affirme à cet effet que

Les œuvres dont le consensus du public littéraire a fait des modèles ou des classiques scolaires peuvent devenir insensiblement les normes esthétiques d'une tradition qui prédéterminera l'attente et l'orientation des générations ultérieures dans le domaines de l'art, (1978:274).

Les parts formelle, esthétique et idéologique de l'œuvre sont dépouillées en vue de favoriser une appropriation assimilation des œuvres littéraires françaises. 


\section{Conclusion}

Au regard de ces différentes données, nous pensons que le rôle dominant joué par la littérature française dans la série littéraire de l'enseignement secondaire congolais n'est pas justifié. Cette littérature occupe une position privilégiée alors que tout porterait à croire que le Congo indépendant allait faire la promotion de sa littérature. Elle se fixe comme la principale réflexion sur la modernité occidentale. II est sans nul doute question de réaffirmer les liens historiques avec l'ancienne puissance coloniale mais une telle politique qui ne dit pas son nom, semble favoriser une culture non nationale. Le métissage culturel émergence mais un métissage qui place le lecteur dans une espèce d'aventure ambiguë. Le défaut de moyens peut justifier le choix d'auteurs français mais l'édification d'une culture nationale devrait nécessairement passer par une mise en exergue de la littérature congolaise. 


\section{Bibliographie}

INRAP (2002). Programme de français des lycées d'enseignement général, Brazzaville, Publication de I'INRAP.

JaUSS Hans Robert (1978). Pour une esthétique de la réception, Paris, Gallimard.

ROBERT Marthe (1977). Livre de lectures, Paris, Grasset, « essais ». 